\title{
Arborescences
}

Revue d'études françaises

\section{Comment débusquer la voix d'un auteur dans sa fiction? Une étude de quelques provocations de Michel Houellebecq}

\section{Raphaël Baroni}

Numéro 6, septembre 2016

Polyphonies : voix et valeurs du discours littéraire

URI : https://id.erudit.org/iderudit/1037505ar

DOI : https://doi.org/10.7202/1037505ar

Aller au sommaire du numéro

Éditeur(s)

Département d'études françaises, Université de Toronto

ISSN

1925-5357 (numérique)

Découvrir la revue

Citer cet article

Baroni, R. (2016). Comment débusquer la voix d'un auteur dans sa fiction? Une étude de quelques provocations de Michel Houellebecq. Arborescences, (6), 72-93. https://doi.org/10.7202/1037505ar

\section{Résumé de l'article}

Michel Houellebecq est un auteur controversé, non seulement parce que certains critiques s'interrogent sur le statut littéraire de son oeuvre, qui a connu un immense succès commercial, mais aussi parce que ses romans, souvent provocateurs, engendrent chez les lecteurs des réactions contrastées, qui vont du rejet radical, accompagné par une mise en cause de la valeur morale du texte, à une forte adhésion, généralement fondée sur le constat de la lucidité de l'auteur et de sa capacité à représenter le monde tel qu'il est, avec férocité, sans complaisance, mais également avec courage et un certain humour. Au coeur de ces jugements qui engagent des valeurs essentielles pour la littérature, se pose la question fondamentale de la polyphonie romanesque. Sur la base d'une analyse de quelques extraits tirés des Particules élémentaires, seront dégagés différents facteurs, textuels et contextuels, susceptibles de conditionner la réception du texte, notamment la perception de la « voix » de l'auteur, tout en offrant un espace ouvert sur une pluralité interprétative. Je me centrerai en particulier sur la manière dont le roman présente des opinions racistes, homophobes et antiféministes susceptibles d'engendrer de violentes réactions de la part du public, tout en engageant, à des degrés différents, la responsabilité morale de l'écrivain. Cette analyse visera également, en préambule, à réfléchir sur le statut éthique des énoncés fictionnels.

Tous droits réservés ( $\odot$ Département d'études françaises, Université de Toronto, Ce document est protégé par la loi sur le droit d’auteur. L’utilisation des 2016 services d'Érudit (y compris la reproduction) est assujettie à sa politique d'utilisation que vous pouvez consulter en ligne.

https://apropos.erudit.org/fr/usagers/politique-dutilisation/ 


\section{SOMMAIRE}

Francis Langevin et Raphaël Baroni.

01 University of British Columbia et Université de Lausanne Introduction

Alain Rabatel. Université de Lyon 1

13 L'énonciation problématisante : en dialogue avec

Le Royaume d'Emmanuel Carrère

Émilie Goin. Université du Luxembourg

39 Analyse d'un discours d'action collective mis en récit.

L'Anarchie dans Les Cloches de Bâle d'Aragon

David Bélanger. Université du Québec à Montréal

54 En contre-jour : la représentation évanescente de l'écrivain dans le roman québécois contemporain

Raphaël Baroni. Université de Lausanne

72 Comment débusquer la voix d'un auteur dans sa fiction?

Une étude de quelques provocations de Michel Houellebecq

Liesbeth Korthals Altes. Rijksuniversiteit Groningen

94 Actes de cadrage, narratologie et herméneutique - à propos de I'indétermination énonciative dans Sujet Angot de Christine Angot

121

Marion Sauvaire et Érick Falardeau. Université Laval

Susciter le moment critique. De l'investissement de ressources axiologiques

Frank Wagner. Université Rennes 2

148 Quand le narrateur boit(e)... (Réflexions sur le narrateur non fiable et/ou indigne de confiance) 


\title{
Comment débusquer la voix d'un auteur dans sa fiction ? Une étude de quelques provocations de Michel Houellebecq
}

\author{
Raphaël Baroni. Université de Lausanne
}

\section{Résumé}

Michel Houellebecq est un auteur controversé, non seulement parce que certains critiques s'interrogent sur le statut littéraire de son cuvre, qui a connu un immense succès commercial, mais aussi parce que ses romans, souvent provocateurs, engendrent chez les lecteurs des réactions contrastées, qui vont du rejet radical, accompagné par une mise en cause de la valeur morale du texte, à une forte adhésion, généralement fondée sur le constat de la lucidité de l'auteur et de sa capacité à représenter le monde tel qu'il est, avec férocité, sans complaisance, mais également avec courage et un certain humour. Au cour de ces jugements qui engagent des valeurs essentielles pour la littérature, se pose la question fondamentale de la polyphonie romanesque. Sur la base d'une analyse de quelques extraits tirés des Particules élémentaires, seront dégagés différents facteurs, textuels et contextuels, susceptibles de conditionner la réception du texte, notamment la perception de la "voix" de l'auteur, tout en offrant un espace ouvert sur une pluralité interprétative. Je me centrerai en particulier sur la manière dont le roman présente des opinions racistes, homophobes et antiféministes susceptibles d'engendrer de violentes réactions de la part du public, tout en engageant, à des degrés différents, la responsabilité morale de l'écrivain. Cette analyse visera également, en préambule, à réfléchir sur le statut éthique des énoncés fictionnels.

\section{Une œuvre polyphonique et ambiguë}

Le potentiel polémique de l'œuvre de Michel Houellebecq révèle une dimension de la littérature qui a été longtemps refoulée par une partie du champ littéraire et de la critique, du moins celle qui se situe dans le sillage de la "mort de l'auteur" décrétée par Barthes dans un article polémique de 1968. Certes Houellebecq n'est pas le seul auteur contemporain à faire tenir des propos scandaleux à ses personnages, voire à ses narrateurs, mais son omniprésence médiatique et sa manière de relayer certaines de ces provocations dans ses propos publics, font que l'autonomie morale dont jouissaient les œuvres de fiction semble avoir volé en éclat. Ainsi que le résume Liesbeth Korthals Altes, les remous provoqués par les romans de Houellebecq ont « ceci de piquant que la question de la position de l'auteur, sinon de ses intentions et de sa morale, si soigneusement évacués par la 
théorie littéraire dominante, [reprennent] tous [leurs] droits» (2004 : 29). En effet, lorsqu'on lit un roman de Houellebecq, il est difficile d'écarter d'emblée la question de la portée morale du livre et de la responsabilité de l'écrivain qui en est l'auteur. Houellebecq s'est d'ailleurs vu assigner plusieurs procès au cours de sa carrière et la publication de son dernier roman, simultanément à l'attentat islamiste contre Charlie Hebdo, le 7 janvier 2015, n'a fait que renforcer cette problématique. Mais le moins que l'on puisse dire, c'est que la manière d'articuler Soumission avec l'actualité politique n'est pas dépourvue d'ambiguïté, de lectures plurielles, voire contradictoires, puisque l'on a pu observer, d'un côté, le Premier ministre français, Manuel Valls, affirmer que « la France ça n’est pas la soumission, la France ça n'est pas Michel Houellebecq, la France ça n'est pas l'intolérance, la haine, la peur, jouer sur les peurs en permanence ${ }^{1}$ ", alors qu'un journaliste allemand affirme, quelques jours plus tard: "Qui voit en Houellebecq un auteur à scandale, qui ne cherche qu'à provoquer, devrait jeter hors de sa bibliothèque Sade, Rimbaud, Balzac ou Baudelaire [...]. Houellebecq est le Charlie Hebdo de la littérature européenne. Je suis Houellebecq ${ }^{2}$ ". On constate ici l'ambivalence des fictions (ou des dessins de presse) qui jouent sur le registre de la provocation : un tel discours réclame une liberté d'expression absolue, mais ne cache pas non plus qu'il défend des valeurs (par exemple la laïcité) ou qu'il vise à dévoiler une réalité occultée par les médias ${ }^{3}$, même au risque d'indigner une partie du public. Le sociologue Éric Fassin ${ }^{4}$ affirme ainsi que Houellebecq revendique pour le roman

tout à la fois l'efficacité et l'irresponsabilité sociales. D'une part, la littérature dit la vérité du monde : elle est la science de notre société. Mais d'autre part, on ne saurait lui demander des comptes, à moins

\footnotetext{
${ }^{1}$ "Manuel Valls: "Ces individus étaient suivis mais il n’y a pas de risque zéro" ", propos recueillis par Yves Calvi et Jean-Michel Apathie, "L'invité de RTL ", RTL, 8 janvier 2015, consulté le 21 décembre 2015, disponible en ligne http://www.rtl.fr/actu/politique/charliehebdo-manuel-valls-sur-rtl-ces-individus-etaient-suivis-mais-il-n-y-a-pas-de-risque-zerosuivez-notre-direct-7776137196.

"Citation tirée de Nathalie Versieux, "Michel Houellebecq triomphe en Allemagne ", Libération, 20 janvier 2015, consulté le 21 décembre 2015, disponible en ligne http://www.liberation.fr/livres/2015/01/20/houellebecq-triomphe-en-allemagne_1184578.

Voir aussi : "Michel Houellebecq encensé en Allemagne ", Le Temps, 20 janvier 2015. La critique en question a été publiée dans le quotidien de gauche Tageszeitung.

${ }^{3} \mathrm{Au}$ sujet de son dernier roman, Houellebecq a affirmé à plusieurs reprises qu'il exprimait les peurs d'une partie importante de la société que les médias, qu’il juge dominés par une idéologie de centre gauche, occulteraient intentionnellement. Ce thème de la dissimulation de la réalité politique par les médias est d'ailleurs au cœur de l'intrigue de Soumission.

${ }^{4}$ Éric Fassin désigne Les particules élémentaires comme le "roman noir de la sexualité française " et affirme que "souvent, les personnages font écho au narrateur, et à l'auteur " (2009: 255).
}

\section{Arborescences}

Revue d'études françaises

ISSN: 1925-5357 
d'être politiquement correct. Comment sceller ce pacte littéraire ambigu ? (Fassin 2009 : 267)

Derrière cette question difficile, qui se pose depuis les débuts littéraires de Houellebecq, et notamment depuis la parution des Particules élémentaires, se profile en fait une problématique très générale liant éthique, polyphonie et auctorialité. Même sans en revenir à la critique biographique à la Lanson, de nombreux théoriciens de la littérature, de Platon à Wayne C. Booth, en passant par Mikhail Bakhtine, ont montré qu'il est impossible de faire l'économie de la figure l'auteur, même si ce dernier n'est qu'un rôle ou une reconstruction imaginaire du lecteur, car sa présence en creux joue un rôle essentiel dans la communication littéraire. Pour Platon, l'auteur camoufle sa voix quand il feint de donner la parole à ses personnages, ce qui le rend suspect dans le jeu démocratique. Bakhtine considère quant à lui que ce n'est qu'avec l'apparition du roman polyphonique, qu'il situe dans l'œuvre de Dostoïevski, que les personnages cessent de n'être que les porte-paroles de l'écrivain. Dès lors, pour Bakhtine, le discours romanesque «offre la singularité d'être bivocal. Il sert simultanément à deux locuteurs et exprime deux intentions différentes : celle - directe - du personnage qui parle, et celle - réfractée - de l'auteur. Pareil discours contient deux voix, deux sens, deux représentations» (Bakhtine 1978: 144). Un tel discours apparaît ainsi comme un « hybride intentionnel » dans lequel

il ne s'agit pas seulement (et pas tellement) du mélange des formes et des indices de deux styles et de deux langages, mais avant tout du choc, au sein de ces formes, des points de vue sur le monde. C'est pourquoi un hybride littéraire intentionnel n'est pas un hybride sémantique abstrait et logique (comme en rhétorique), mais concret et social. (Bakhtine 1978 : 177)

C'est également sur le terrain des valeurs et de l'éthique que Wayne C. Booth (1977) a élaboré pour sa part la notion d'auteur implicite, qui lui sert à évaluer la distance relative qui peut exister entre le lecteur, les personnages, le narrateur et l'image de l'auteur que se reconstruit le lecteur. Ainsi que l'affirme Booth, l'auteur " peut être à une plus ou moins grande distance des autres personnages - ceci allant de l'approbation sans réserve que Jane Austen manifeste pour Jane Fairfax dans Emma, à son mépris pour Wickham dans Orgueil et Préjugés"(1977: 104). Il ajoute que "ce que nous appelons "engagement" ou "sympathie" ou "identification" est généralement fait d'un ensemble de réactions vis-à-vis des auteurs, des narrateurs, des observateurs et des autres personnages " (1977: 104). La notion de "narrateur non-fiable » apparaît comme un cas de figure dans lequel le lecteur est capable d'entendre, derrière le discours explicite d'un personnage, l'ironie implicite de l'auteur, qui communique en quelque sorte par-dessus son épaule. Booth précise que 
de telles situations "exigent beaucoup plus de perspicacité de la part du lecteur que ne l'exige une narration en laquelle on se fie au narrateur » (1977 : 106).

Plus récemment, dans son dernier ouvrage, Liesbeth Korthals Altes (2014) a proposé quant à elle de refonder l'analyse narratologique des textes littéraires en adoptant une approche "méta-herméneutique " fondée sur l'analyse des opérations interprétatives mobilisées par des lecteurs empiriques, en ménageant une place centrale à l'attribution de valeurs, ce qui débouche sur la nécessité de replacer au cœur de l'enquête la manière dont la figure de l'auteur est inscrite en creux dans ses fictions. Elle s'inspire notamment, en plus des références à l'approche de Booth, des travaux de Jérôme Meizoz (2004; 2007 ; 2011), qui a montré que l'auteur se sert de ses œuvres pour construire un ethos qui rejaillit sur son image publique, mais également, dans une sorte de retour de balancier, que cette image publique peut apparaître comme une "posture" susceptible de transformer la perception de son œuvre.

Ces approches ont en commun de considérer que la figure de l'auteur est une dimension incontournable du phénomène littéraire ${ }^{5}$, ce qui l'engage sur un plan éthique, mais en même temps, elles soulignent que cette "voix auctoriale » se trouve prise dans un enchevêtrement de voix alternatives qui en complexifient le statut.

Certes, le retour de la figure de l'auteur dans l'œuvre de Houellebecq accompagne une tendance plus large, elle s'inscrit notamment dans le sillage de Doubrovsky, d'Ernaux et de tant d'autres qui accompagnent l'essor de l'autofiction en France. Cependant, Houellebecq s'en distingue par sa tendance à mettre au premier plan un discours apparemment sérieux tenu sur le monde, la société et la nature humaine, là où d'autres écrivains semblent surtout intéressés par l'exposition de leur égo. Il s'ensuit que la portée éthique de ce discours apparaît beaucoup plus problématique et conduit à s'interroger sur le caractère assumé des assertions fictionnelles. Dans un tel contexte, de nombreux commentateurs ont fondé leur analyse en niant purement et simplement les frontières qui séparent traditionnellement les opinions de l'auteur de celles exprimées par ses personnages, que ce soit pour le critiquer ou, au contraire, pour célébrer son génie visionnaire. Dans Houellebecq économiste, par exemple, Bernard Maris analyse finement les réflexions exposées par Houellebecq dans ses romans, mais il procède comme si l'auteur s'était exprimé en son nom propre, dans le registre de l'essai. Il ne s'embarrasse donc pas de préciser qu'en tel endroit, c'est tel personnage qui affirme telle chose dans telles circonstances, et que, par conséquent, l'auteur

${ }^{5}$ Par "phénomène ", j'entends la rencontre du texte et du lecteur, car même si l'auteur est formellement absent de l'œuvre, le lecteur a besoin de son image, ainsi que le reconnaît Barthes dans ses derniers travaux (1973). 
ne partage pas nécessairement ce point de vue. Il est cependant difficile de critiquer la démarche de Maris quand on se situe à un tel niveau de généralité, tant la vision économique qui se dégage de l'ensemble des romans apparaît cohérente, ce qui suggère qu'elle est le produit d'une réflexion unique qu'il est difficile de ne pas rattacher à une vision personnelle de l'auteur et à un discours sérieux sur le monde.

Cet écrasement de la polyphonie romanesque que l'on observe dans certains commentaires n'est cependant pas exempt d'ambiguïtés. Ainsi que le souligne Bruno Viard, si le lecteur a l'impression d'entendre la "voix " de Houellebecq, il ne sait pas nécessairement à quelle «facette » de l'écrivain il a affaire :

Le mystère Houellebecq, c'est qu'il existe deux Houellebecq, un méchant Houellebecq, le mieux connu du grand public, provocateur qui dépasse plus souvent qu'à son tour la limite du tolérable, qui profère des énormités d'un air de ne pas y toucher, qui choque par trop le respect dû aux gens. Et un gentil Houellebecq, qui parle d'amour et de bonté, qui prend la défense des enfants délaissés, des filles moches et des vieillards abandonnés. Lire Houellebecq, c'est écouter ces deux voix narratives si opposées, au lieu de n'écouter que celle qu'on préfère, et tenter d'interpréter une contradiction aussi patente et aussi dérangeante. (Viard $2013: 12-13$ )

D'autres chercheurs, dans le sillage de Bakhtine, soulignent néanmoins l'importance de tenir compte d'une polyphonie plus fondamentale, qui ne tient pas à la dualité de l'auteur, mais à celle de la scène d'énonciation particulière du roman. Ainsi, Samuel Estier se demande si cette pluralité de voix ne vient pas simplement

de la confusion entre un Houellebecq qui serait philosophe et le Houellebecq romancier, à savoir que le roman comme genre, au sens où l'entend Bakhtine, entretient un rapport aux idées différent de celui de l'essai. "La démarche polyphonique est incompatible avec un monologisme de type habituel " (Bakhtine 1970 : 25), écrivait Bakhtine pour marquer la singularité du roman quant à la portée des idées qui y sont défendues et contestées par une pluralité de voix. (Estier 2013: $\$ 17)$

Liesbeth Korthals Altes, dans sa lecture des Particules élémentaires, conclut son analyse de cet " étrange roman à thèse » dans le même sens :

Ce roman possède certes des traits du roman à thèse, notamment le fort appel à l'adhésion du lecteur. Mais il n'en présente guère l'univocité : parodie, incongruité et humour minent pour le lecteur sensible à ces signaux, toute cristallisation en une doctrine. Ils invitent au contraire à 
une connivence avec l'auteur, même si la position affective et idéologique de celui-ci reste finalement insaisissable, oscillant entre des registres incompatibles - ironique, grotesque et désespéré. (Korthals Altes $2004: 43$ )

Cette propriété ne tient pas uniquement à la dimension fictionnelle du roman, mais également à sa dimension narrative puisque, ainsi que l'affirme Alain Rabatel,

le fait même de raconter, en mettant en scène des centres de perspective différents, ouvre potentiellement une boîte de Pandore d'où sortent des voix autorisées et d'autres qui le sont moins, mais qui néanmoins sapent l'autorité des premières, en sorte que le récit, loin d'être l'illustration d'une vérité préétablie, ouvre sur les possibles infinis de l'interprétation. (Rabatel $2008: 17$ )

Reste que Houellebecq, en flirtant parfois avec le registre de l'autofiction ${ }^{6}$, mais aussi en reprenant à son compte publiquement, parfois littéralement, certains propos tenus par ses personnages ${ }^{7}$, ou encore en encourageant le public à lire son œuvre "au premier degré $e^{8}$, a lui-même contribué à rendre poreuses les frontières qui séparent les opinions des personnages de celles de l'auteur, au risque de renforcer l'écrasement de la polyphonie. Il n'en continue pas moins de défendre l'autonomie relative de ses personnages, qu'il définit comme des parasites :

\footnotetext{
${ }^{6}$ Certes, Houellebecq fait plutôt partie des écrivains qui opèrent un "retour au réel ", renonçant ainsi à un certain narcissisme de leurs contemporains, mais l'écrivain se sert de sa biographie pour rendre crédible sa vision du monde, pour l'ancrer dans une expérience authentiquement vécue. Les traces autofictionnelles peuvent concerner autant le nom complet de l'auteur (qui est un personnage de La Carte et le territoire), ou le prénom de certains personnages ou narrateurs, que certains éléments de leur biographie, effet particulièrement visible dans les récits d'enfance de Bruno et Michel dans Les particules élémentaires.

${ }^{7}$ Le cas le plus emblématique reste l'affirmation, dans un entretien publié en 2001 dans le magazine Lire, que "la religion la plus con, c'est quand même l'Islam ». Jérôme Meizoz souligne que ces propos semblent reprendre les paroles d'un personnage de Plateforme, à la différence près que, dans la fiction, Aïcha qualifie avec cet adjectif « des personnes, alors que Houellebecq désigne une religion : la critique porte sur une idée, et n'est pas judiciable du même jugement " (Meizoz 2004 : 197).

8 "Le spectacle de la société ", Les Inrockuptibles, 14 mars 2000. Dans cet entretien entre Bret Easton Ellis et Michel Houellebecq repris à Der Spiegel, Houellebecq affirme : " on ne prend pas suffisamment mes livres au premier degré » (cité par Fassin 2009 : 265). Il a récemment réaffirmé ce désir d'une lecture au premier degré sur le plateau de l'émission On n'est pas couchés, diffusée le 29 juillet 2015.
}

\section{Arborescences}

Revue d'études françaises

ISSN: 1925-5357 
C'est comme faire une expérience où tu nourrirais des parasites, des créatures dans ton cerveau où tu les laisses se développer. Au départ, une dose de soi leur assure une bonne viabilité, mais après il faut les laisser vivre... ou les supprimer s'ils prennent le dessus. Les personnages sont des vampires, ils veulent absolument exister. (Houellebecq et Kapriélan $2010: 43)$

Les personnages se trouvent ainsi écartelés entre leur fonction de porte-parole de l'écrivain et leur valeur proprement mimétique, qui consiste à produire l'illusion qu'il s'agit de personnes ayant une existence autonome et possédant leur propre voix. Mais Vincent Jouve a montré que cette dualité correspond surtout à deux attitudes de lecture : le lectant appréhende "le personnage comme un instrument entrant dans un double projet narratif et sémantique» alors que le lisant le considère plutôt comme une "personne évoluant dans un monde dont lui-même participe, le temps de la lecture " (Jouve 1992 : 110). Lors d'une récente interview entre Houellebecq et Pujadas (qui se trouve être également un personnage de Soumission, dont le narrateur loue les qualités professionnelles), le contraste entre ces deux attitudes interprétatives apparaît clairement. Alors que le journaliste affirme que les lecteurs - désignés par le pronom inclusif " on " - sont enclins à s'interroger sur le rapport entre les opinions de l'auteur et celles des personnages, Houellebecq se contente d'affirmer que ces derniers doivent être surtout convaincants, et que l'auteur ne doit pas les juger :

Pujadas : Il y a une question simple qu'on se pose en lisant le livre. Ce nouvel ordre religieux, la hiérarchie homme-femme, et ce que vous... tout ce que vous décrivez, le retour de la polygamie, est-ce que vous l'approuvez? Est-ce que vous approuvez votre héros lorsqu'il se convertit?

Houellebecq : Euh, ni l'un ni l'autre.

Pujadas: C'est ce qu'on sent dans le livre mais c'est troublant.

Houellebecq : Oui, c'est troublant, je sais bien, mais il y a une espèce de relativisme généralisé, qui s'empare du personnage et de l'auteur aussi à la suite, quoi ! On... c'est vrai qu'il faut...

Pujadas : Et ce relativisme, vous le partagez !

Houellebecq : Oui, je sais pas, je sais même plus. Mais faut pas juger les gens quand on écrit. Enfin, il faut que tous les personnages aient raison. C'est-à-dire, celui qui le convertit à l'Islam, le professeur d'université qui s'est lui-même converti, il est important qu'il soit séduisant et 
convaincant tant qu'il parle. Ça ne veut pas dire que je l'approuve, mais il faut qu'il soit séduisant et convaincant. Et il l'est.'

On constate ainsi l'existence de tensions entre différents lecteurs, ou du moins entre différentes attitudes interprétatives, mais aussi entre certains lecteurs et la position défendue par l'écrivain. Ces tensions gravitent autour de la question de savoir où se situe l'auteur, de quelle manière il habite sa fiction, comment sa voix se fait entendre à côté de ou, au contraire, à travers celle de ses personnages, entre ironie et discours délégué, entre polyphonie et symphonie. Il faut prendre ici le mot « voix » dans son sens éthique, à l'instar de Mieke Bal qui souligne que " pour rendre compte que le récit ne vient pas de nulle part et que quelqu'un en est responsable, le concept [de voix] paraît indispensable» (Bal 2001 : 9). Dans la même perspective, il faut comprendre la "polyphonie» comme une énigme, bien davantage que comme une technique narrative :

la question " qui » n'est pas seulement une personnification, sans doute problématique, d'un aspect textuel, mais aussi un début d'enquête, une question whodunnit, qui a commis ce crime, qui indique que s'il y a paroles qui portent, qui font acte comme nous l'a appris la théorie des actes du langage, il y a responsabilité pour ces actes. (Bal 2001 :9-10)

Certes, il y a des auteurs qui ont joué la carte du retrait le plus radical possible, tant publiquement que dans la scénographie de leurs romans, ces derniers ne prétendant pas renvoyer à autre chose qu'à eux-mêmes. Mais pour les autres, en particulier pour Houellebecq, qui met en scène publiquement ses opinions, qui choisit de s'incarner dans ses fictions et qui reconnaît que ses romans devraient être pris au sérieux, la situation est beaucoup plus délicate. Pour l'auteur, il y a alors deux façons de se disculper: nier le statut performatif des actes de langage fictionnels ou prétendre n'être que le transcripteur objectif d'une réalité qui le dépasse. Sur le premier plan, qui s'appuie sur la spécificité de la fiction comme texte à visée esthétique, Houellebecq affirme dans le même entretien avec Pujadas qu'il ne connaît pas "d'exemples où un roman aurait changé le cours de l'histoire. C'est autre chose qui change le cours de l'histoire. C'est des essais, le Manifeste du parti communiste, des choses comme ça, mais pas des romans".

Sur le second plan, il soutient que la vision du monde que l'on trouve dans ses romans n'est qu'un compte-rendu objectif de la réalité, et que s'il s'inspire de sa propre vie, ce n'est que pour dégager une réalité qui transcende le cas individuel. Le point essentiel n'est plus, dès lors, de maintenir une

9 Transcription du journal télévisé de France 2, le 5 janvier 2015, trois jours avant la parution du livre. URL : https://www.youtube.com/watch?v=8E-lkVp8oHY\&feature=youtu.be. 
distinction entre les opinions des personnages et celles de l'auteur, mais de soutenir que ces individus (fictifs et réel) incarnent et exemplifient une réalité sociale qui les englobe ou une vérité humaine qui les traverse. Ainsi, Houellebecq s'oppose à ce qu'il appelle une "réduction de la littérature au témoignage " et il espère à l'inverse une lecture sociologisante ou philosophique, au-delà de cette singularisation. C'est ce qu'il affirme avec force dans son essai épistolaire Ennemis publics, rédigé peu après la publication de la «biographie non autorisée » de Demonpion :

Il y a depuis l'origine quelque chose, dans ma littérature, qui a partie liée avec la honte. Je m'attendais à vrai dire, en publiant mes premiers livres, à susciter sur ma personne une certaine honte [...]. Ce qui m'attendait au contraire, la merveilleuse surprise, c'est que des lecteurs sont venus à moi pour me dire: "Mais non, ce que vous décrivez ce sont des choses humaines, certaines humaines en général, d'autres spécifiques à l'être humain des sociétés humaines occidentales contemporaines... Nous vous sommes reconnaissants, au contraire, d'avoir eu le courage de dévoiler ces choses, d'avoir pris sur vous cette part de honte..."

C'est cela, je pense, que l'on n'a pas supporté. Et l'on a eu de cesse d'établir que mes livres n'étaient nullement l'expression d'une vérité humaine générale, mais celle d'un traumatisme individuel; et dans ce combat acharné la biographie, la minable et sotte biographie, est bel et bien l'arme la plus efficace, l'offensive ayant atteint, ces dernières semaines, son point le plus élevé. (Houellebecq et Levi 2008 : 241)

L'argument est donc le suivant: lire au premier degré ne conduit pas nécessairement à entendre la voix de l'écrivain en tant qu'individu, car ce dernier est capable de se faire le porte-voix d'une génération ou d'une époque, de s'élever en adoptant le point de vue impersonnel du sociologue ou celui, intemporel, du philosophe. L'auteur se fait donc entendre, mais il s'affranchit par le haut de sa biographie individuelle. Ce qu'il puise dans sa vie pour construire ses personnages n'est que ce qui ne lui appartient pas en propre, ce qu'il juge exemplaire d'une génération, d'un genre ou de l'humanité.

Cette dissociation explique la manière dont l'auteur des Particules élémentaires s'incarne à la fois dans les personnages de Michel, témoin neutre d'un monde dans lequel il ne s'engage pas, et dans celui de Bruno, personnage qualifié de "représentatif de son époque» (Houellebecq, Les Particules élémentaires ${ }^{10}$ : 63), d' «élément passif du déploiement d'un mouvement historique" (Houellebecq, Les Particules élémentaires: 178). Bruno, tout comme Houellebecq, est un être dont les "champs de forces " qui structurent sa « conscience et ses désirs " appartiennent "à l'ensemble de sa génération "

${ }^{10}$ La pagination se réfère à l'édition de poche " J'ai lu », imprimée en 2007. 
(Houellebecq, Les particules élémentaires: 178). Mais Houellebecq en tant qu'écrivain se juge également capable, à l'instar de Michel, de percevoir ces champs de forces et de les transposer dans ses romans, aussi objectivement que possible. C'est ainsi qu'il serait à la fois légitime d'entendre l'auteur et illégitime de s'en tenir à cette seule voix individuelle, qui ne rendrait pas justice aux ambitions du romancier. Ainsi que le résume Pierre Jourde :

Un personnage n'est pas son auteur, mais une figure possible de sa personnalité, une potentialité qu'il a plus ou moins développée dans la réalité. "Ce qu'on pourrait être et qu'on n'est pas ", dit justement Houellebecq dans Lire. En faisant le portrait de Bruno, ou de Michel dans Plateforme, à la fois assumés et refusés, Houellebecq met en jeu le raciste en lui. Au lieu de montrer un méchant raciste, il laisse s'exprimer, prendre corps une part malsaine de lui-même. Il la met en jeu, ce qui signifie qu'il la met en question, qu'il la soumet à l'analyse. Il fait son travail de romancier. (Jourde $2002: 274$ )

Reste que Houellebecq reconnaît, sur ce point, que le combat est "acharné " et que certains lecteurs continuent de réduire ses romans à un témoignage individuel, ce qui les conduit à demander à l'auteur d'assumer la responsabilité des discours tenus par ses personnages, car rien, au fond, ne garantit que cette histoire individuelle soit véritablement exemplaire et que la vision du monde véhiculée par le livre soit neutre et objective. Répondre à cette question dépend non seulement de la "perspicacité " du lecteur, qui le conduit à entendre les nuances d'un discours dans lequel les voix de l'auteur et celles des personnages peuvent être en consonance ou, au contraire, entrer en dissonance, mais cela met également en jeu les valeurs idéologiques et la vision du monde de l'interprète, qui doit encore décider s'il accepte de partager les "points de vue sur le monde " de cet "hybride intentionnel " auquel il est confronté, et donc juger si ce dernier incarne une vérité qui le transcende, une vérité que le lecteur serait prêt à faire sienne.

\section{L'auteur des Particules élémentaires est-il responsable des opinions provocantes exprimées par ses personnages?}

Dans cette partie, je vais tenter d'éclairer le fonctionnement discursif de quelques passages représentatifs des provocations que l'on trouve dans Les particules élémentaires, de manière à éclairer le type de postures interprétatives 
qu'ils sont susceptibles d'engendrer ${ }^{11}$. En première approximation, on peut poser l'hypothèse que les passages qui s'écartent de manière ostensible d'une idéologie "bien-pensante" placent le lecteur face à une alternative : soit les assertions sont prises au sérieux - auquel cas l'auteur sera tenu responsable des opinions exposées par le texte -, soit elles sont considérées comme ironiques ou humoristiques. Dans le cas où l'auteur est pris au sérieux, la question sera alors de savoir si la provocation permet de dévoiler une vérité générale, ou si elle doit être réduite à une singularité biographique, qui permettrait de mettre en cause l'auteur sur un plan éthique.

Le commentaire des Particules élémentaires par Jose Domingues de Almeida apparaît comme un cas exemplaire de la première option. Selon lui, le fréquent recours à ce qu'il désigne comme un "commentaire épiphrastique ${ }^{2}$ " (Almeida 2007: 187) ferait entendre "la voix abjecte et sournoise de l'auteur" (Almeida 2007 : 187). Le choix des adjectifs marque d'emblée le refus par le lecteur de partager la vision du monde rattachée à la figure de l'écrivain. Almeida souligne par ailleurs que Houellebecq exprimerait notamment ses opinions sur la " femme libérée », qui « fait l'objet de virulentes attaques misogynes " (Almeida 2007 : 187) ; le pamphlet raciste de Bruno permettrait à l'écrivain "de le signer sans signature explicite " (Almeida 2007: 188). Almeida ajoute enfin que le "narrateur/auteur/personnage " décrit les homosexuels "selon les attentes et les préjugés de l'écrivain" (Almeida 2007: 188). Derrière ces opinions, Almeida reconnaît par ailleurs que l'écrivain défend sérieusement une thèse : "c'est surtout le procès de la liberté des mœurs, acquis majeur du Mai 68 français, [...] qui est imputé à l'outrageant Houellebecq" (Almeida 2007 : 182). Mais si le roman met en scène, sur un plan sociologique et historique, la " crise du mâle» dans la société contemporaine, Almeida précise, par un jeu de parenthèses, que ce sujet générique, étendard d'une génération, dissimule en fait un sujet beaucoup plus individuel :

\footnotetext{
${ }^{11}$ Je ne me poserai pas directement la question de savoir si la provocation est un simple artifice commercial ou si elle remplit une finalité esthétique. Sur ces questions, Jean-François Patricola dans Houellebecq ou la provocation permanente (2005) considère qu'il s'agit d'une stratégie commerciale de l'écrivain, alors qu'Éric Fassin (2009 : 268) raisonne plus finement sur le lien entre provocation et littérarité en soulignant le rôle joué par la figure de Sollers dans le dispositif des Particules élémentaires.

${ }^{12}$ Bien qu'il ne définisse pas exactement ce qu'il entend par "commentaire épiphrastique», l'analyse fait ressortir des passages dans lesquels une voix impersonnelle adopte un ton "documentaire» ou "entomologique» (Almeida 2007 : 187). Le Littré définit l'épiphrase comme une figure de style "par laquelle on ajoute, à une phrase qui semblait finie, un ou plusieurs membres pour développer des idées accessoires». On pourrait rattacher cette figure, dans le cas du discours houellebecquien, à ce que nous désignerons plus bas de "registre aphoristique ".
} 
Ce mâle (ou cet écrivain) aura tendance à traiter les femmes soi-disant

libérées de toutes sortes d'attributs péjoratifs ou assassins. Puisqu' " on assiste en fait au retournement de certaines théories féministes extrêmes" (Lindenberg, 2002: 24), ce mâle dominant (écrivain et homme frustré) n'hésitera pas à réduire la femme à la condition animale de la femelle au sein du troupeau ». (Almeida 2007 : 182)

Almeida, conclut néanmoins son analyse par un constat ambivalent :

Notre aperçu du discours tenu par l'auteur et les personnages de Les particules élémentaires fait apparaître des apories de la société française contemporaine, et a la capacité de confondre le lecteur. Ce lecteur s'est montré plutôt séduit par l'insolence des romans houellebecquiens, mais sans trop savoir s'il est invité, en clin d'œil, à endosser les propos qui y sont émis dans l'épiphrase et le poncif. (Almeida 2007 : 188)

Adoptant une perspective très platonicienne, cette lecture souligne donc le pouvoir manipulateur de la fiction, sa "capacité à confondre le lecteur", en faisant de chaque discours assumé par un personnage fictif un levier argumentatif dans une communication biaisée, parce que le locuteur réel avance masqué. Dans une lecture assez semblable, Pierre Jourde considère cependant que "Houellebecq est provocateur, mais prudent" (Jourde 2002 : 272) et qu'il «joue habilement sur la différence entre auteur, narrateur et personnage " (Jourde 2002 : 273). Il précise que

Tous les propos ouvertement racistes ou antimusulmans sont tenus par des personnages secondaires ou, dans Les Particules élémentaires, par le demi-frère de Michel, Bruno. [...] Dans l'hypothèse idéale, Houellebecq nous montrerait comme un individu peut devenir raciste, comment ce racisme peut prendre naissance dans ses problèmes sexuels, son malheur, sa frustration afin de mieux condamner ce processus. [...] Trop facile, et faux, comme le montrent les convergences entre fiction et propos privés. (Jourde $2002: 273$ )

Pour faire la part des choses, il convient donc de commencer l'analyse en observant la manière dont l'auteur construit la scène énonciative des passages les plus provocateurs de son roman ${ }^{13}$, en scrutant notamment la manière dont il parvient à atténuer la portée de certains propos, alors que, dans d'autres cas, il apparaît plus enclin à assumer le point de vue de tel ou tel personnage. Commençons par citer in extenso trois passages caractéristiques de ces provocations qui mettent en cause des opinions respectivement racistes, homophobes et antiféministes :

${ }^{13}$ Dominique Maingueneau décrit cette opération sous le nom de « scénographie » (2004). 
[1] Quoi qu'il en soit, dans les douches du Gymnase Club j'ai pris conscience que j'avais une toute petite bite. J'ai vérifié chez moi : 12 centimètres, peut-être 13 ou 14 en tirant au maximum le centimètre pliant vers la racine de la bite. J'avais découvert une nouvelle source de souffrance; et là il n'y avait rien à faire, c'était un handicap radical, définitif. C'est à partir de ce moment que j'ai commencé à haïr les nègres. Enfin il n'y en avait pas beaucoup au lycée, la plupart étaient au lycée technique Pierre-de-Coubertin, là même où l'illustre Defrance faisait du striptease philosophique et de la lèche pro-jeunes. Il y en avait juste un dans mes classes, en première $A$, un grand costaud qui se faisait appeler Ben. Il était toujours avec une casquette et des Nike, je suis sûr qu'il avait une bite énorme. Évidemment, toutes les filles étaient à genoux devant ce babouin; et moi qui essayais de leur faire étudier Mallarmé, ça n'avait aucun sens. C'est comme ça que devait finir la civilisation occidentale, me disais-je avec amertume, se prosterner à nouveau devant les grosses bites, tel le babouin hamadryas. (Houellebecq, Les particules élémentaires : 191-192)

[2] Il s'installa à l'écart et n'engagea la conversation avec personne ; en mastiquant ses céréales vitaminées il songeait au vampirisme de la quête sexuelle, à son aspect faustien. C'est tout à fait faussement, pensait par exemple Bruno, qu'on parle d'homosexuels. Lui-même n'avait jamais, ou pratiquement jamais, rencontré d'homosexuels; par contre, il connaissait de nombreux pédérastes. Certains pédérastes - heureusement peu nombreux - préfèrent les petits garçons; ceux-là finissent en prison, avec des peines de sûreté incompressibles, et on n'en parle plus. La plupart des pédérastes, cependant, préfèrent les jeunes gens entre quinze et vingt-cinq ans; au-delà il n'y a plus, pour eux que de vieux culs flapis. Observez deux vieilles pédales entre elles, aimait à dire Bruno, observez-les avec attention : parfois il y a une sympathie, voire une affection mutuelle; mais est-ce qu'elles se désirent? en aucun cas. Dès qu'un petit cul rond de quinze - vingt-cinq ans vient à passer, elles se déchirent comme deux vieilles panthères sur le retour, elles se déchirent pour posséder ce petit cul rond; voilà ce que pensait Bruno.

Comme en bien d'autres cas, les prétendus homosexuels avaient joué un rôle de modèle pour le reste de la société, pensait encore Bruno. Luimême, par exemple, avait quarante-deux ans ; désirait-il pour autant les femmes de son âge? En aucune façon. Par contre, pour une petite chatte enrobée dans une minijupe, il se sentait encore prêt à aller jusqu'au bout du monde. Enfin du moins jusqu'à Bangkok. Treize heures de vol tout de même. (Houellebecq, Les particules élémentaires: 105-106)

[3] J'ai jamais pu encadrer les féministes... reprit Christiane alors qu'ils étaient à mi-pente. Ces salopes n'arrêtaient pas de parler de vaisselle et de partage des tâches; elles étaient littéralement obsédées par la vaisselle. Parfois elles prononçaient quelques mots sur la cuisine ou les

\section{Arborescences}


aspirateurs ; mais leur grand sujet de conversation, c'était la vaisselle. En quelques années, elles réussissaient à transformer les mecs de leur entourage en névrosés impuissants et grincheux. À partir de ce moment - c'était absolument systématique - elles commençaient à éprouver la nostalgie de la virilité. Au bout du compte, elles plaquaient leurs mecs pour se faire sauter par des machos latins à la con. J'ai toujours été frappée par l'attirance des intellectuelles pour les voyous, les brutes ou les cons. Bref, elles s'en tapaient deux ou trois, parfois plus pour les très baisables, puis elles se faisaient faire un gosse et se mettaient à préparer des confitures maison avec les fiches cuisines Marie Claire. J'ai vu le même scénario se reproduire, des dizaines de fois. (Houellebecq, Les particules élémentaires : 182-183)

Sur un plan lexical, ce qui frappe d'emblée dans les trois extraits, c'est la vulgarité du registre (" bite ", " con », «baisable », etc.) ainsi que l'usage de termes possédant des connotations problématiques (" nègres", "pédales ", "salopes»). Sur ce point, il n'est pas inutile de noter que deux de ces extraits se rattachent au personnage de Bruno, qui concentre dans le roman les passages les plus vulgaires, alors que son frère Michel ou le narrateur impersonnel qui prend en charge le récit cadre apparaissent beaucoup plus proches du point de vue de l'auteur implicite et adoptent généralement un registre neutre, détaché, voire scientifique. Les provocations "lexicales " sont ainsi, en quelque sorte, déléguées à des personnages secondaires ou à Bruno, dont le rôle essentiel est d'exemplifier l'état de la société occidentale à la fin $\mathrm{du} \mathrm{XX}^{\mathrm{e}}$ siècle.

Les extraits [2] et [3] possèdent en outre des caractéristiques qui les distinguent assez nettement de l'extrait [1], car ils se rattachent peu ou prou au registre de l'aphorisme ou de ce que l'on pourrait appeler un discours de vérité générale. Ce registre se manifeste, sur un plan linguistique, par l'usage du présent ou d'un imparfait à valeur intemporelle, ainsi que d'articles ou de pronoms à valeur générique: texte [2]: "La plupart des pédérastes, cependant, préfèrent les jeunes gens entre quinze et vingt-cinq ans "; "Comme en bien d'autres cas, les prétendus homosexuels avaient joué un rôle de modèle pour le reste de la société »; texte [3] : "À partir de ce moment c'était absolument systématique - elles commençaient à éprouver la nostalgie de la virilité ». La conclusion du troisième extrait insiste sur le caractère invariant de la trajectoire de vie des féministes, qui est racontée sur le mode de l'imparfait à valeur itérative : "J'ai vu le même scénario se reproduire, des dizaines de fois ». Par définition, ces énoncés sont détachables de leur contexte et les assertions apparaissent dès lors transférables à un nombre indéfini de situations ou d'existants, dans et hors de la fiction. Ainsi que l'a montré Vladimir Dolezel :

La sémantique de ces digressions est déterminée par le fait qu'elles prétendent être valides dans le monde réel; par conséquent, elles sont 
sujettes aux conditions de vérité propres aux textes factuels. Le lecteur ou l'interprète (qui ressent habituellement que ces digressions expriment les opinions propres de l'auteur) a le droit de demander si elles sont vraies ou fausses dans le monde réel. (Dolezel 1998: 27, je traduis)

On constate donc que de tels passages, même s'ils ne mettent pas directement en cause Houellebecq, nourrissent le soupçon que ces digressions pourraient refléter les opinions de l'écrivain. En revanche, on ne trouve rien de tel dans l'extrait [1], le seul article défini à valeur générique étant placé en position d'objet, évitant ainsi qu'un prédicat ne lui soit associé : "j'ai commencé à haïr les nègres" est équivalent à "j'ai jamais pu encadrer les féministes ", mais contrairement à [2], cette assertion n'est pas suivie d'énoncés explicatifs du type « ils étaient... ». La conclusion de [1] semble relever de l'aphorisme, mais elle ne concerne pas les Noirs, mais l'ensemble de la civilisation occidentale, qui est comparée à une société de babouins "hamadryas". Il me semble que ce premier extrait a donc un statut beaucoup moins problématique que les deux autres, dans la mesure où il apparaît difficile de l'associer à une opinion de l'écrivain sur les Noirs. Ou plus exactement, s'il y a généralisation possible, elle concerne plutôt le cas pathologique que représente Bruno, et non l'objet de sa haine, qui n'est motivée que par une cause indirecte, liée à sa frustration.

$\mathrm{Du}$ point de vue de l'ethos des trois personnages qui énoncent ces discours, on peut également remarquer de profondes différences, notamment entre les extraits [1] et [3]. Il faut prendre ici le terme ethos dans son sens rhétorique originel: il ne s'agit pas de l'image de l'auteur projetée par la fiction, mais de l'image de l'énonciateur qui sert d'argument d'autorité pour convaincre un auditoire. Là où Bruno apparaît comme un personnage symptomatique, dont la déchéance met à nu la frustration et l'égoïsme qui caractérisent sa génération, Christiane est au contraire un personnage généreux, connoté positivement tout au long du roman et dont le destin tragique la place plutôt dans un rôle de victime de la société. On peut donc la considérer comme un énonciateur idéologiquement plus fiable que Bruno, alors même que son identité la situe à une plus grande distance «biographique » de l'écrivain. Par ailleurs, la déclaration raciste de Bruno est complètement décrédibilisée par ce qui la fonde. Bruno n'a pas été victime de violences raciales, il ne manifeste pas davantage de connaissances particulières sur les Africains ou sur leurs cultures, il est simplement jaloux d'un attribut qu'il ne possède $\operatorname{pas}^{14}$. Si le racisme est présenté par l'auteur, et par Bruno luimême, comme la conséquence d'une frustration sexuelle, on voit mal comment on pourrait prendre au sérieux tout argument qui serait tenu à

${ }^{14}$ Évidemment, le fait d'attribuer à tous les Noirs un phallus imposant relève du stéréotype raciste que le roman n'invite pas à déconstruire, car il le considère comme un présupposé. 
l'encontre des Noirs sur cette base. À l'inverse, ce n'est pas un hasard si le pamphlet antiféministe, qui rejoint l'un des thèmes centraux du roman, est énoncé par une femme, qui de surcroît souligne à plusieurs reprises que son discours est étayé par une expérience concrète et répétée : "j’ai toujours été frappée "; "c cétait absolument systématique "; "J'ai vu le même scénario se reproduire, des dizaines de fois». Sur ce point, il est évident que l'antiféminisme de l'auteur apparaît à travers le choix d'une énonciatrice présentée comme digne de confiance ${ }^{15}$, alors que l'on ne peut pas conclure grand-chose des propos tenus par Bruno sur les Noirs.

Sur le plan de la prise en charge énonciative, on peut aussi constater que les passages [1] et [3] sont des monologues en style direct enchâssés dans le récit principal, alors que le texte [2] recourt au discours indirect introduit, qui se réfere soit aux pensées de Bruno, soit à des discours antérieurs. Dans ce cas, qui est le plus complexe du point de vue énonciatif, il y a un risque que le lecteur confonde la source de l'énoncé avec celui d'un narrateur impersonnel, qui pourrait être lui-même confondu avec ce qu'Almeida désignait comme un " commentaire épiphrastique " faisant entendre la "voix abjecte et sournoise de l'auteur ». On remarque cependant l'abondance des énoncés de prise en charge énonciative qui neutralisent une telle interprétation : «il songeait »; " pensait par exemple Bruno »; " aimait à dire Bruno »; "voilà ce que pensait Bruno"; "pensait encore Bruno". Cette prise en charge, qui semble excessive pour un passage aussi court, trahit probablement le besoin de l'auteur de prendre ses distances par rapport à des assertions qu'il ne pourrait pas tenir sérieusement, notamment l'affirmation particulièrement choquante affirmant que tous les homosexuels sont en réalité des pédérastes. Mais audelà de cette provocation, qui repose sur un cliché homophobe, la thèse sur laquelle débouche le raisonnement de Bruno - à savoir que les homosexuels joueraient " un rôle de modèle pour le reste de la société » en étant en avance sur leur temps - pourrait certainement être soutenue sérieusement par l'auteur, et tenue pour vraie par certains lecteurs. Ainsi que l'affirme Éric Fassin,

L'homosexualité [...] apparaît essentiellement comme la pointe avancée de la consommation hédoniste : l'auteur l'évoque surtout pour son rôle moteur dans la double valorisation de la taille du pénis (PÉ : 238) et de la jeunesse des corps (PÉ : 131) - deux thèses obsédantes chez Houellebecq. (Fassin 2009 : 258-259)

${ }^{15}$ De manière similaire, ainsi que le note Jérôme Meizoz, les critiques contre l'Islam dans Plateforme émanent de locuteurs appartenant au monde musulman mais qui en ont rejeté ses principes religieux : "énoncée fictivement de l'intérieur, la critique gagne en légitimité " (2004: 193). 
En cela, un lecteur averti devrait être capable de faire la part des choses entre une provocation gratuite, qui bascule rapidement dans le registre du grotesque - notamment lorsque Bruno décrit "deux vieilles pédales [qui] se déchirent comme deux vieilles panthères sur le retour [pour] posséder ce petit cul rond" - et un discours sérieux qui se rattache à la thèse principale développée par Houellebecq dans son roman, et de roman en roman : la société occidentale post-soixante-huitarde soumet les relations amoureuses aux mêmes règles régissant l'économie de marché, la jeunesse et les capacités de séduction étant devenues des valeurs absolues dans une compétition sexuelle généralisée.

Face à une telle analyse, est-il encore nécessaire de s'interroger sur l'éventuelle homophobie de Houellebecq et de faire son procès moral ? Si tel était le cas, alors il faudrait commencer par comparer ce passage avec d'autres opinions exprimées par d'autres personnages ou par l'auteur lui-même. Toutes ces voix parlent-elles à l'unisson ? Si l'on se prête à une telle étude, même à l'échelle de ce seul roman, on constate rapidement que les représentations de l'homosexualité sont beaucoup plus diverses que le point de vue provocateur exprimé par Bruno. Sans quitter le cliché, le personnage de Michel entrevoit par exemple l'homosexualité comme une vie possible, marquée par une solidarité et un engagement qui lui font défaut :

De son côté Michel vivait dans un monde précis, historiquement faible, mais cependant rythmé par certaines cérémonies commerciales - le tournoi de Roland-Garros, Noël, le 31 décembre, le rendez-vous bisannuel des catalogues 3 Suisses. Homosexuel, il aurait pu prendre part au Sidathon, ou à la Gay Pride. Libertin, il se serait enthousiasmé pour le Salon de l'érotisme. Plus sportif, il vivrait à cette même minute une étape pyrénéenne du tour de France. (Houellebecq, Les particules élémentaires: 122)

Le personnage de Desplechin évoque quant à lui un homosexuel victime de l'idéologie conservatrice : "J'ai perdu de vue Philippe après le bac, mais j'ai appris qu'il s'était suicidé quelques années plus tard. Enfin, je ne pense pas que ce soit lié : être à la fois homosexuel, catholique intégriste et royaliste, ça ne doit quand même pas être un mélange très simple" (Houellebecq, Les particules élémentaires: 271). Difficile par ailleurs de débusquer des propos concernant l'homosexualité dans les prises de parole publiques de l'auteur, sauf à prendre en compte son goût affiché pour les scènes pornographiques mettant en scène des lesbiennes. Bref, si le recyclage de stéréotypes fait partie de son dispositif romanesque, la provocation ne dépasse guère la plaisanterie graveleuse, bien que le discours de Bruno sur les homosexuels serve indirectement une argumentation beaucoup plus sérieuse.

On peut également chercher à mettre au jour les opinions de l'auteur en ce qui concerne le racisme et le féminisme en prolongeant l'analyse 
textuelle par une étude faisant intervenir non seulement le cotexte, mais également l'intertextualité, qui inclut aussi bien les autres romans de Houellebecq - qui permettent de faire émerger des thématiques récurrentes imputables à l'auteur - que ses prises de parole dans l'espace public. Certes, lorsque Sollers commente le pamphlet raciste de Bruno, il est question du désir de " redevenir des animaux, des animaux dotés d'une grosse bite et d'un tout petit cerveau reptilien, annexe à leur bite " (Houellebecq, Les particules élémentaires: 195), mais là encore, le ton humoristique du passage - réaction de Sollers : "Vous êtes authentiquement raciste, ça se sent, ça vous porte, c'est bien. Boum, boum !» (Houellebecq, Les particules élémentaires : 195) - et le caractère non fiable du personnage qui rédige ce pamphlet, font que l'ironie de l'auteur se fait entendre de manière assez évidente. D'ailleurs, c'est l'ensemble des personnages qui sont abordés sous l'angle de leur animalité, Michel et Annabelle étant par exemple décrits comme des mammifères soumis aux lois de la nature. Par ailleurs, on ne trouve pas, à ma connaissance, de propos ouvertement racistes dans les déclarations publiques de Houellebecq, à part cette allusion, qui fait beaucoup penser à la vision cynique de l'Afrique que partageait en son temps François Mitterrand : "Bien sûr qu'il y a des victimes dans les conflits du tiers monde, mais ce sont elles qui les provoquent. Si ça les amuse de s'étriper, ces pauvres cons, qu'on les laisse s'étriper ${ }^{16} »$. Certes, cette indifférence est d'une froideur monstrueuse, mais elle trahit surtout un manque d'intérêt pour les problématiques du tiersmonde. D'ailleurs, à l'échelle de l'œuvre entière, il apparaît que la question des races ne joue qu'un rôle marginal, les romans étant plutôt centrés sur des problématiques économiques, sexuelles ou religieuses. D'ailleurs, dans son dernier roman (Houellebecq, Soumission, 2015), les seules allusions à des Français d'origine africaine placent ces derniers en position d'adjuvants du protagoniste ou de victimes innocentes des changements politiques décrits par la fiction, les forces véritablement inquiétantes étant incarnées par les extrémistes religieux et les nationalistes d'extrême droite :

En débouchant place d'Italie, je fus soudain envahi par la sensation que tout pouvait disparaître. Cette petite Noire aux cheveux bouclés, au cul moulé dans un jean, qui attendait le bus 21, pouvait disparaître ; elle allait certainement disparaître, ou du moins être sérieusement rééduquée. (Houellebecq, Soumission: 90)

Ce n'est que vers huit heures et demie qu'un vigile fit son apparition, venant du secrétariat principal, et se posa derrière les grilles pour nous informer que la fac était fermée toute la journée, et le resterait jusqu’à

${ }^{16}$ Propos recueillis dans Lire, cités par Jourde (2002 : 268). François Mitterrand, face à la tragédie rwandaise, a affirmé quant à lui qu' « un génocide dans ces pays-là, ce n’est pas très important " (propos rapportés par Patrick de Saint-Exupéry cités dans Le Figaro du 12 janvier 1998). 
nouvel ordre. Il ne pouvait pas nous en dire plus; nous devions rentrer chez nous, nous serions "informés individuellement ". C'était un Noir bonhomme, un Sénégalais si je me souviens bien, que je connaissais depuis des années, et que j'aimais bien. (Houellebecq, Soumission : 118119)

En revanche, sur le front de l'antiféminisme, les opinions de l'auteur sont beaucoup plus facilement audibles et elles se dévident comme un véritable fil d'Ariane tout au long de sa carrière. Bien que changeant de locuteurs (Christiane, Bruno, Michel, Annabelle, le narrateur impersonnel), le roman accumule des aphorismes qui parlent à l'unisson pour décrire des différences sexuelles jugées naturelles. Bruno: "avoir un enfant aujourd'hui, n'a plus aucun sens pour un homme. Le cas des femmes est différent, car elles continuent à éprouver le besoin d'avoir un être à aimer - ce qui n'est pas, ce qui n'a jamais été le cas des hommes" (Houellebecq, Les particules élémentaires: 169). Annabelle : "Les hommes ne font pas l'amour parce qu'ils sont amoureux, mais parce qu'ils sont excités ; cette évidence banale, il m’a fallu des années pour la comprendre" (Houellebecq, Les particules élémentaires : 233). Il faut noter que sur le plan de la connotation idéologique, l'opposition entre hommes et femmes semble toujours fonctionner au profit de ces dernières, de sorte qu'il apparaît difficile de confondre l'antiféminisme de Houellebecq avec une forme de misogynie. Ainsi que le souligne Éric Fassin,

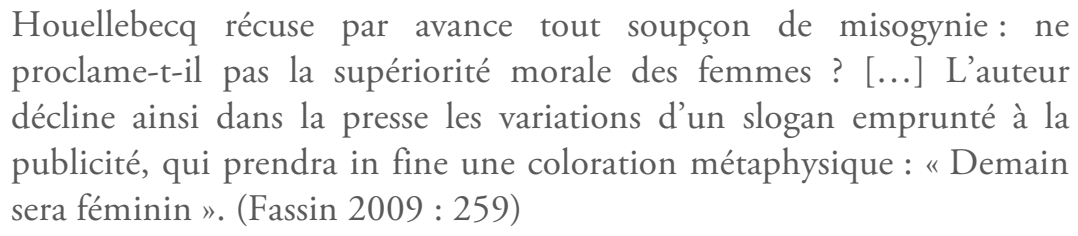

Il n'en reste pas moins que cette opposition des genres fige en nature des différences que certaines féministes récusent, tout en critiquant leurs objectifs d'émancipation et d'égalité entre les sexes. Houellebecq est d'ailleurs allé jusqu'à afficher ouvertement ses opinions dans le registre de l'essai, à l'occasion d'une postface au Scum Manifesto de Valerie Solanas :

Ainsi pouvait-on dans les années soixante-dix les voir lutter pour la contraception, l'avortement, la liberté sexuelle, etc. tout à fait comme si le "système patriarcal " était une invention des méchants mâles, alors que l'objectif historique des hommes était à l'évidence de baiser le maximum de nanas sans avoir à se mettre une famille sur le dos. Les pauvres poussaient même la naïveté jusqu'à s'imaginer que l'amour lesbien, condiment érotique apprécié par la quasi-totalité des hétérosexuels en activité, était une dangereuse remise en cause du pouvoir masculin. Elles manifestent enfin, et c'était le plus triste, un 
incompréhensible appétit à l'égard du monde professionnel et de la vie de l'entreprise ; les hommes, qui savaient depuis longtemps à quoi s'en tenir sur la «liberté » et l' "épanouissement" offerts par le travail, ricanaient doucement. (Houellebecq 2005 : 92-93)

Dans ce texte, il est facile d'identifier non seulement la vision du monde de Valérie, mais également son style pamphlétaire, directement assumé par l'auteur, mais cette fois son ethos spécifique lui permet de déconstruire les valeurs féministes en dévoilant le point de vue des "méchants mâles ", leurs "objectifs historiques", leurs fantasmes érotiques ainsi que leurs « ricanements». On constate ainsi que les rapprochements que l'on pouvait établir, à partir d'une analyse purement textuelle et énonciative, entre la voix de l'écrivain et celle de son porte-parole fictif, se trouvent ainsi largement confirmés par la lecture complète du roman et de l'œuvre, qui apparaissent en définitive, sur cette question, beaucoup moins polyphoniques que symphoniques. Et cette symphonie antiféministe rejoint également la voix propre de l'auteur lorsqu'il s'exprime hors du registre fictionnel, le contexte médiatique contribuant d'autant plus à conditionner la lecture. Dans un tel cas, la polyphonie n'est qu'un simple vernis, une condition nécessaire de l'assertion feinte, qui ne cherche même pas à dissimuler l'évidence, à savoir qu'il s'agit également d'une assertion sérieuse de l'auteur.

\section{Conclusion}

Il ne fait guère de doute que les romans les plus provocateurs sont également ceux qui sont les plus aptes à induire chez les lecteurs un questionnement concernant les opinions de l'écrivain, les conduisant parfois sur le seuil d'une interprétation qui nie les distinctions traditionnelles entre assertions sérieuses et assertions feintes, et au sein de ces dernières, qui atténue les distinctions traditionnelles entre les voix de l'auteur, du narrateur et des personnages. J'ai essayé de montrer que le degré d'implication de l'auteur n'est cependant pas uniforme suivant le type de provocation, les trois extraits que j'ai commentés permettant d'observer une gradation fondée sur des scénographies différenciées. Il ne suffit donc pas de mettre en rapport le discours d'un personnage avec le point de vue de l'écrivain qui a écrit ce discours, mais il faut encore compter sur la perspicacité d'un lecteur sensible à la dimension polyphonique du discours pour moduler son jugement moral en fonction de facteurs textuels ou contextuels. Dans cette interprétation oscillant entre polyphonie et symphonie, la scénographie locale doit être mise en relation avec son cotexte, l'intertexte (notamment l'éventuelle lecture des autres romans du même écrivain) et le contexte médiatique dans lequel l'auteur affiche, ou refuse d'afficher, ses opinions personnelles. C'est l'ensemble de ces facteurs qui concourent à produire un équilibre fragile dans 
une interprétation partiellement indéterminée, parce que cette dernière est également largement conditionnée par les valeurs propres du lecteur. Ainsi que l'affirme Francis Langevin :

cette polyphonie éthique [...] peut difficilement rester purement "littéraire" ou "artistique ", et par conséquent se contenter de l'indécidabilité - après tout pas si surprenante en littérature contemporaine: elle transporte au contraire le texte et ses enjeux internes sur le terrain du lecteur, de ses valeurs, et de toutes les interactions discursives que permet cette sortie du littéraire [...]. (Langevin $2014: 108$ )

Il est dès lors toujours possible, même pour un lecteur perspicace, c'est-à-dire celui qui comprend l'effet visé par la scénographie, de juger le texte sur une base purement éthique et personnelle, de refuser par exemple de disculper un auteur qui prétendrait se contenter de représenter le monde "tel qu'il est " (alors que ce monde pourrait être, est peut-être, autre) ou simplement de refuser ses provocations gratuites, c'est-à-dire de refuser la connivence du sourire en coin lorsqu'un cliché transgresse les normes de la bien-pensance. Même lorsque l'auteur lorgne en direction du lecteur par-dessus l'épaule du personnage, ce lecteur est libre de détourner le regard.

\section{Références bibliographiques}

De Almeida, J. D. 2007. "Réactions à la réaction. Brèves considérations sur le sens de l'épiphrase dans Les particules élémentaires de Michel Houellebecq ». Cédille 3 : 179-190.

Bakhtine, M. 1978. Esthétique et théorie du roman. Paris: Gallimard.

Bal, M. 2001. «Voix/voie narrative : la voix métaphorée ». Cahiers de narratologie 10 (1) : 9-36. URL : http://narratologie.revues.org/6909. Page consultée le 29 mai 2015.

Baroni, R. 2014. "La guerre des voix : critique polyphonique et divergences interprétatives dans l'œuvre de Michel Houellebecq ". COnTEXTES. URL : http://contextes.revues.org/5979. Page consultée le 21 décembre 2015.

Barthes, R. 1984. "La mort de l'auteur". Dans Le bruissement de la langue. Essais critiques IV. Paris : Seuil : 63-69. [1968].

Barthes, R. 1973. Le plaisir du texte. Paris : Seuil.

Booth, W. C. 1977. "Distance et point de vue ». Dans Poétique du récit, sous la direction de G. Genette et T. Todorov. Paris : Seuil : 85-113 [1961].

Dolezel, L. 1998. Heterocosmica: Fiction and Possible Worlds. Baltimore: Johns Hopkins University Press.

Estier, S. 2013. " L'énigme Houellebecq ". Acta fabula 14 (7). URL : http://www.fabula.org/revue/document8188.php. Page consultée le 21 décembre 2015.

Fassin, É. 2009. "Le roman noir de la sexualité française". Dans Le sexe politique. Genre et sexualité au miroir transatlantique. Paris : Éditions de l'EHESS : 251-268.

Houellebecq, M. 1998. Les particules élémentaires. Paris : Flammarion.

\section{Arborescences}


Houellebecq, M. 2005. "L'humanité, second stade ». Dans SCUM Manifesto. Paris: Fayard: 91-101.

Houellebecq, M. 2015. Soumission. Paris : Flammarion.

Houellebecq, M. et N. Kapriélian. 2010. Les Inrockuptibles $771: 36-43$.

Houellebecq, M. et B.-H. Lévy. 2008. Ennemis publics. Paris : Flammarion et Grasset.

Jourde, P. 2002. "L'individu louche: Michel Houellebecq". Dans La littérature sans estomac. Paris : L'esprit des Pénoncules : 265-289.

Jouve, V. 1992. "Pour une analyse de l'effet-personnage ». Littérature 85 : 103-111.

Korthals Altes, L. 2004. "Persuasion et ambiguïté dans un roman à thèse postmoderne (Les Particules élémentaires)". Dans Michel Houellebecq, sous la direction de S. Van Wesemael. Amsterdam et New York : Rodopi : 29-45.

Korthals Altes, L. 2014. Ethos and Narrative Interpretation: The Negociation of Values in Fiction. Lincoln et Londres : University of Nebraska Press.

Langevin, F. 2014. "Genre, géographie et héritage : lecture éthique du style du narrateur dans L'annonce de Marie-Hélène Lafon ». Tangence 105 : 91-108.

Maingueneau, D. 2004. Le discours littéraire. Paratopie et scène d'énonciation. Paris: Armand Colin.

Maris, B. 2014. Houellebecq économiste. Paris : Flammarion.

Meizoz, J. 2004. «Le roman et l'inacceptable. Sociologie d'une polémique : autour de Plateforme de Michel Houellebecq ". Dans L'ail sociologue et la littérature. Genève: Slatkine Érudition : 181-209.

Meizoz, J. 2007. Postures littéraires. Genève : Slatkine Érudition.

Meizoz, J. 2011. La fabrique des singularités. Postures littéraires II. Genève : Slatkine Érudition.

Patricola, J.-F. 2005. Houellebecq ou la provocation permanente. Paris : Écriture.

Rabatel, A. 2008. Homo narrans. Limoges : Éditions Lambert-Lucas.

Schaeffer, J.-M. 1994. "Le récit fictif ». Dans Modernité, fiction, déconstruction, ouvrage dirigé par J. Bessière. Paris : Lettres Modernes.

Searle, J. 1975. "The Logical Status of Fictional Discourse." New Literary History 6 (2) : 319332.

Viard, B. 2013. Les tiroirs de Michel Houellebecq. Paris : Presses Universitaires de France. 